\title{
Pericarditis and myocarditis after COVID-19 mRNA vaccination in a nationwide setting
}

Jonathan Yap ${ }^{1,2}{ }_{M B B S}$, Mun Yee $\underline{\text { Tham }}{ }^{3}{ }_{M P H}$, Jalene $\underline{\text { Poh }}{ }^{3}{ }_{B S C}$, Dorothy $\underline{T o h}^{3}{ }^{3} h D$, Cheng Leng $\underline{\text { Chan }}{ }^{3}$ PhD, Toon Wei Lim ${ }^{4}{ }_{M B B S}$, Shir Lynn $\underline{\operatorname{Lim}}{ }^{4}{ }_{M B B S}$, Yew Woon $\underline{\text { Chia }}{ }^{5}{ }_{M B B S}$, Yean Teng $\underline{\operatorname{Lim}}{ }^{6}{ }_{M B B S}$, Jonathan $\underline{\text { Choo }}{ }^{7}{ }_{M B B S}$, Zee Pin Ding ${ }^{1,2}{ }_{M B B S}$, Ling Li $\underline{\text { Foo }}^{8}{ }_{P h D}$, Simin $\underline{K u O}^{8}{ }_{B S S}$, Yee How Lau ${ }^{1}{ }_{B S c}$, Annie Lee ${ }^{1}{ }_{P h D}$, Khung Keong Yeo ${ }^{1,2}{ }_{M B B S}$

\begin{abstract}
Introduction: Despite reports suggesting an association between COVID-19 mRNA vaccination and pericarditis and myocarditis, detailed nationwide population-based data are sparsely available. We describe the incidence of pericarditis and myocarditis by age categories and sex after COVID-19 mRNA vaccination from a nationwide mass vaccination programme in Singapore.

Methods: The incidence of adjudicated cases of pericarditis and myocarditis following COVID-19 mRNA vaccination that were reported to the vaccine safety committee between January to July 2021 was compared with the background incidence of myocarditis in Singapore.

Results: As of end July 2021, a total of 34 cases were reported (9 pericarditis only, 14 myocarditis only, and 11 concomitant pericarditis and myocarditis) with 7,183,889 doses of COVID-19 mRNA vaccine administered. Of the 9 cases of pericarditis only, all were male except one. The highest incidence of pericarditis was in males aged 12-19 years with an incidence of 1.11 cases per 100,000 doses. Of the 25 cases of myocarditis, $80 \%$ ( 20 cases) were male and the median age was 23 years (range 12-55 years) with 16 cases after the second dose. A higher-than-expected number of cases were seen in males aged 12-19 and 20-29 years, with incidence rates of 3.72 and 0.98 case per 100,000 doses, respectively.

Conclusion: Data from the national registry in Singapore indicate an increased incidence of pericarditis and myocarditis in younger men after COVID-19 mRNA vaccination.
\end{abstract}

Ann Acad Med Singap 2022;51:96-100

Keywords: COVID-19 vaccine, myocarditis, pericarditis

\section{INTRODUCTION}

The coronavirus disease 2019 (COVID-19) pandemic has resulted in much morbidity and mortality worldwide. The development of mRNA vaccines has heralded much hope in the fight against the disease. The safety and efficacy of these vaccines have been well demonstrated in clinical trials, ${ }^{1,2}$ and also against severe disease from variants. $^{3}$

With the implementation of mass nationwide vaccination programmes across many countries, rarer vaccination-related adverse events not seen in clinical trials have surfaced. In particular, recent reports have indicated an association between COVID-19 mRNA vaccination and pericarditis and myocarditis, especially in younger males. ${ }^{4-7}$ However, detailed nationwide population-based data remain lacking.

We aim to study the incidence of pericarditis and myocarditis by age bands and sex after COVID-19 mRNA vaccination from a nationwide mass vaccination programme in Singapore.

\footnotetext{
${ }^{1}$ Department of Cardiology, National Heart Centre Singapore, Singapore

${ }^{2}$ Duke-NUS Medical School, Singapore

${ }^{3}$ Health Sciences Authority, Singapore

${ }^{4}$ Department of Cardiology, National University Heart Centre, Singapore

${ }^{5}$ Department of Cardiology, Tan Tock Seng Hospital, Singapore

${ }^{6}$ Mount Elizabeth Hospital, Singapore

${ }^{7}$ Department of Paediatrics, KK Women's and Children's Hospital, Singapore

${ }^{8}$ National Registry of Diseases Office, Health Promotion Board, Singapore

Correspondence: Clin A/Prof Khung Keong Yeo, Department of Cardiology, National Heart Centre Singapore, 5 Hospital Drive, Singapore 169609.

Email: yeo.khung.keong@singhealth.com.sg
} 


\section{CLINICAL IMPACT}

\section{What is New}

- Data from Singapore indicate an increased incidence of pericarditis and myocarditis among men below 30 years old.

- The absolute incidence of pericarditis and myocarditis remained low and most cases responded well to treatment.

\section{Clinical Implications}

- The risk of pericarditis and myocarditis following COVID-19 mRNA vaccination should be balanced against the risk of developing severe COVID-19 infection.

\section{METHODS}

\section{Study population}

Singapore is an Asian city-state with a population of about 5.45 million people. The Pfizer-BioNTech COVID-19 mRNA vaccine was first rolled out in the country end of December 2020, followed by the Moderna COVID-19 mRNA vaccine in March 2021. The vaccination programme kicked off for those above 70 years old from January 2021, followed by subsequent age groups. Vaccination was made available to those aged 12-39 years old from 21 June.

As of end July 2021, there were about 65,000 COVID-19 positive cases in Singapore with 37 confirmed COVID-related deaths. The Health Sciences Authority (HSA) is the national regulatory authority in Singapore that actively monitors the safety of these vaccines. Adverse events suspected to be associated with these vaccines are reported by healthcare professionals to HSA. Reports of myocarditis and pericarditis are reviewed and adjudicated using the US Centers for Disease Control and Prevention (CDC) definition of myocarditis and pericarditis by an independent expert panel of cardiologists appointed by HSA. ${ }^{8}$ Confirmed and probable cases based on the above definition were included. ${ }^{8}$ This study is a retrospective review of HSA vaccine adverse event reporting system up to 25 July 2021. Waiver of ethics approval for this study was obtained from the SingHealth institutional review board.

\section{Other data sources and outcomes}

Demographic and clinical data available from the HSA vaccine adverse event reporting system were also obtained. These included age, sex, type and dose number of vaccine, onset duration and length of hospital stay.

The National Immunisation Registry (NIR) keeps a comprehensive record of all COVID-19 vaccinations administered to the population of Singapore. Aggregate data on the number of COVID-19 mRNA vaccines doses by age bands and sex were obtained from NIR to calculate incident rates of events per 100,000 vaccine doses. Background population rates of myocarditis (per 100,000 population/year) by age bands and sex from the last decade prior to COVID-19 vaccination were also obtained from the Ministry of Health to allow for comparison with incident rates.

\section{Statistical analysis}

Baseline characteristics of study patients were summarised as frequencies and percentages for categorical variables and median \pm range/interquartile range (IQR) for continuous variables. Incident rates and expected cases were calculated for the whole cohort as well as by age bands and sex. Incident rates were calculated by dividing number of incident cases by the number of doses of vaccines administered for the particular category multiplied by 100,000 to give the incident rates per 100,000 vaccine doses. Expected cases were calculated by multiplying the respective background rates of myocarditis by the total number of vaccine doses given for the particular category, adjusting for a time period of 30 days after vaccination dose.

\section{RESULTS}

As of 25 July 2021, a total of 34 cases were reported (9 pericarditis only, 14 myocarditis only, and 11 with concomitant pericarditis and myocarditis) (Fig. 1). This was against the backdrop of 7,183,889 doses $(1,298,117$ one dose only and 2,942,886 two doses) of COVID-19 mRNA (both Pfizer-BioNTech and Moderna) administered. The breakdown of the distribution of the vaccination by age band are as follows: 12-19 (524,051 doses), 20-29 (1,081,953 doses), 30-39 (1,357,293 doses), 40-49(1,461,617 doses), 50-59(1,151,414 doses), and 60 and above $(1,607,561$ doses $)$.

\section{Pericarditis}

Of the 9 cases of pericarditis only, all were males except for one. The median age was 31 years (range 14-53 years) with 3 cases (all males) below 20 years. Six cases were after the first dose and 3 cases were after the second dose. The median time of onset after vaccination was 2 days (IQR 1-2 days). Six cases required hospitalisations with a median length of stay 
of 2.5 days (IQR $2-4$ days). One case (53 years old) required intensive care management. There were no mortalities.

The highest incidence of pericarditis only was in males aged 12-19 years with an incidence of 1.11 cases per 100,000 doses. For the 10-year age bands from 20 to 59 years for males, the incident rates were fairly similar ranging from $0.13-0.26$ case per 100,000 doses. There were no cases in those 60 years old and above (Table 1 and Fig. 1B).

\section{Myocarditis}

There were 25 cases of myocarditis (12 confirmed, 13 probable), of which 11 had concomitant pericarditis (Fig. $1 \mathrm{C}$ ). Of these, $80 \%$ ( 20 cases) were male and the median age was 23 years (range $12-55$ years) with 16 cases (all males) below age 30 years. Nine cases were after the first dose and 16 cases were after the second dose. The median time of onset after vaccination was 3 days (IQR $1-5$ days). All cases required hospitalisations with a median length of stay of 3 days (IQR $2-5$ days). One ( 16 years old) required intensive care management. There were no mortalities.

Table 1 shows a higher-than-expected number of cases were seen in males aged $12-19$ and 20-29 years, with incidence rates of 3.72 and 0.98 cases per 100,000 doses, respectively. Fairly similar incidence rates of $0.35-0.42$ case per 100,000 doses were seen in males aged 30-39 and females aged 40-49 and 50-59. There were no cases in those 60 years old and above.

\section{DISCUSSION}

To our knowledge, this is first large nationwide population-based study in Singapore on the incidence of pericarditis and myocarditis following mRNA COVID-19 vaccination, with detailed breakdown by age bands and sex, and independently adjudicated outcomes. Several pertinent observations were made: (1) there was an increased incidence of pericarditis and myocarditis in males below 30 years old with a higher incidence in those even younger (less than 20 years old); (2) the absolute incidence remained low and most cases responded well to treatment; (3) there were no reported cases in females below 40 years old nor among all above 60 years old.

In a report of more than 2.8 million doses of mRNA COVID-19 vaccine administered in the US military, a higher-than-expected rate of 0.82 myocarditis case per 100,000 doses of vaccine was observed. ${ }^{4}$ Notably, $87 \%$ of these cases were after the second dose. Although sex and age comparisons may be more difficult to infer from a military study, all were male with a median age of 25 years old. The US CDC also reported a rate of 0.35 myocarditis case per 100,000 second doses of mRNA COVID-19 vaccine with the highest rate in males aged 18-29 (2.43 cases per 100,000 second doses). ${ }^{5}$ There were no confirmed myocarditis associated deaths. In a recently published nationwide Israeli study, mRNA COVID-19 vaccination resulted in about 3 excess myocarditis cases per 100,000 persons. ${ }^{6}$ Among the 21

Table 1. Incidence of pericarditis and myocarditis by age band

\begin{tabular}{|c|c|c|c|c|c|c|c|}
\hline & \multicolumn{7}{|c|}{ Incidence per 100,000 vaccine doses by age band (years) } \\
\hline & $12-19$ & 20-29 & 30-39 & $40-49$ & $50-59$ & 60 and above & All ages \\
\hline \multicolumn{8}{|c|}{ Pericarditis and/or myocarditis } \\
\hline Male & 4.83 & 1.14 & 0.65 & 0.27 & 0.17 & 0 & 0.75 \\
\hline Female & 0 & 0 & 0 & 0.56 & 0.35 & 0 & 0.17 \\
\hline Overall & 2.48 & 0.65 & 0.37 & 0.41 & 0.26 & 0 & 0.47 \\
\hline \multicolumn{8}{|c|}{ Pericarditis only } \\
\hline Male & 1.11 & 0.16 & 0.26 & 0.13 & 0.17 & 0 & 0.21 \\
\hline Female & 0 & 0 & 0 & 0.14 & 0 & 0 & 0.03 \\
\hline Overall & 0.57 & 0.09 & 0.15 & 0.14 & 0.09 & 0 & 0.13 \\
\hline Male & 3.72 & 0.98 & 0.39 & 0.13 & 0 & 0 & 0.53 \\
\hline Female & 0 & 0 & 0 & 0.42 & 0.35 & 0 & 0.15 \\
\hline Overall & 1.91 & 0.55 & 0.22 & 0.27 & 0.17 & 0 & 0.35 \\
\hline
\end{tabular}




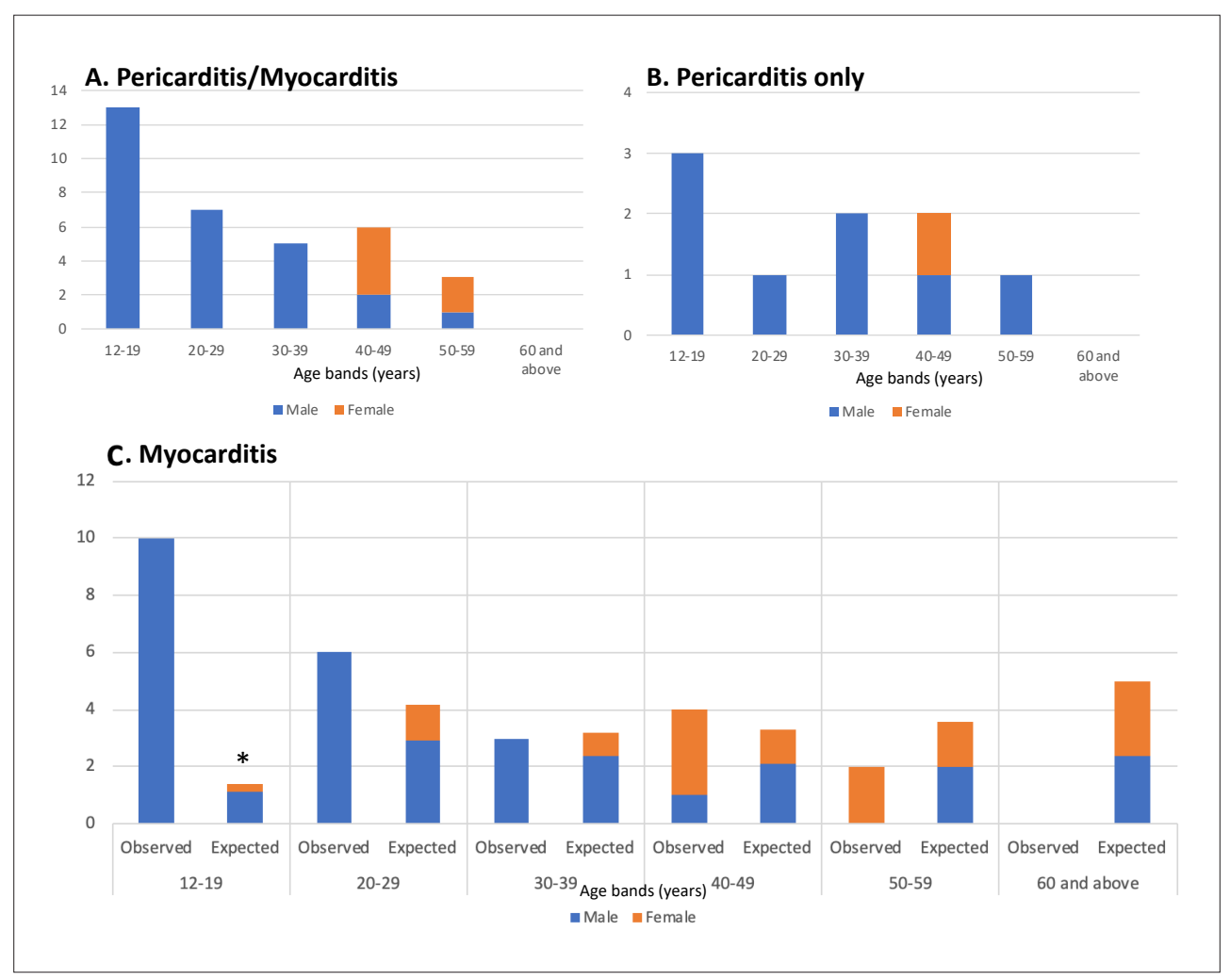

Fig. 1. Nationwide cases of pericarditis and/or myocarditis cases in Singapore by age band and sex. (A) Pericarditis and/or myocarditis cases. (B) Pericarditis only cases. (C) Expected and observed myocarditis cases (*age band 10-19).

persons with myocarditis in the study, the median age was 25 years (IQR 20-34), and 90.9\% were male. Surveillance reports from the Israeli Ministry of Health found a potential link between the second vaccine dose and the myocarditis among males aged 16-30 years old, with a stronger link in those aged 16-19 years old. 95\% of the cases were mild. ${ }^{9}$ Our study adds further knowledge to this field. We found an above expected rate of myocarditis primarily in younger males, with the highest incidence in those aged 12-19 (3.72 cases per 100,000 doses) followed by 20-29 (0.98 case per 100,000 doses). Similarly, majority of the cases happened after the second dose, but responded well to treatment and have been discharged from hospital.

With regards to pericarditis, a prior study from the US showed about $60 \%$ of the 37 cases occurred after the second dose. ${ }^{10}$ In this study, two-thirds of the 9 cases occurred after the first dose. The small numbers in our study preclude meaningful conclusion and will be better elucidated with further data collection.

Several possible pathophysiological mechanisms have been put forward to explain the development of myocarditis after COVID-19 mRNA vaccination. ${ }^{11}$ These include the triggering of an exaggerated response by the immune system to the mRNA vaccine in susceptible individuals, resulting in a proinflammatory cascade..$^{11,12}$ Similarities between the spike protein of the COVID-19 virus used in mRNA vaccines and self-antigens may result in autoimmune reactions. ${ }^{11,13}$ The preponderance of young males may be explained by the more active immune system in the young. Sex hormone differences have shown that testosterone is known to inhibit antiinflammatory cells while oestrogen inhibits proinflammatory T cells..$^{11,14}$

Despite the increased incidence in younger males, the absolute incidence was low at $<5$ cases per 100,000 doses across multiple studies. ${ }^{4-6,9}$ In the aforementioned Israeli study, ${ }^{6}$ while vaccination resulted in an elevated risk of myocarditis at 2.7 events per 100,000 persons, COVID-19 infection itself was associated with a substantially increased risk of myocarditis (11 cases per 100,000). ${ }^{6}$ With COVID-19 infections being endemic and with the more virulent nature of certain variants (such as Alpha and Delta), the risks of vaccination have to be balanced against the possibility of severe COVID-19 disease as well as the greater public health 
implications for spread. Based on risk-benefit assessment, the US Advisory Committee on Immunization Practices has continued to recommend COVID-19 vaccination with any Food and Drug Administration-approved vaccine for those $\geq 18$ years and the Pfizer-BioNTech COVID-19 mRNA vaccine for those $\geq 12$ years. ${ }^{5}$ In Singapore, the Ministry of Health has also recommended COVID-19 vaccination for those above 12 years old. ${ }^{15}$ The recommendation for vaccination in those above 12 years old has also been put forth in various other publications. ${ }^{11}$ Of note, recent data have shown an increased risk of pericarditis/myocarditis with the Moderna vaccine when compared to the Pfizer-BioNTech vaccine. In a large Danish population-based study, a significantly increased risk of myopericarditis was found, driven by an increased risk among those aged $12-39$ years. ${ }^{16}$ This has led to several Nordic countries restricting the use of the Moderna vaccine in the younger age groups. The differential risk of myopericarditis by vaccine type will be the work for further research.

\section{Limitations}

Cases not reported to the HSA may have been missed but the extent of under-reporting for adverse events is expected to be low as there is an established robust framework for reporting that is well adhered to by healthcare professionals in Singapore. Additional clinical data on the cases of myocarditis and pericarditis were not available for the purposes of the present study. Nevertheless, the reported cases were independently adjudicated by an expert committee of cardiologists, who had the required comprehensive clinical data available.

\section{CONCLUSION}

In the setting of a comprehensive nationwide vaccination programme in Singapore, an increased incidence of pericarditis and myocarditis was noted predominantly in younger males after COVID-19 mRNA vaccine administration. However, the majority responded well to treatment and the absolute incidence remained very low. The decision for vaccination has to be balanced against the potential of severe adverse clinical outcomes and public health implications with actual COVID-19 infection.

\section{REFERENCES}

1. Polack FP, Thomas SJ, Kitchin N, et al. Safety and efficacy of the BNT162b2 mRNA Covid-19 vaccine. N Engl J Med 2020;383:2603-15.

2. Baden LR, El Sahly HM, Essink B, et al. Efficacy and safety of the mRNA-1273 SARS-CoV-2 vaccine. N Engl J Med 2021; 384:403-16.

3. Lopez Bernal J, Andrews N, Gower C, et al. Effectiveness of Covid-19 vaccines against the B.1.617.2 (Delta) variant. N Engl J Med 2021;385:585-94.

4. Montgomery J, Ryan M, Engler R, et al. Myocarditis following immunization with mRNA COVID-19 vaccines in members of the US military. JAMA Cardiol 2021;6:1202-6.

5. Rosenblum HG, Hadler SC, Moulia D, et al. Use of COVID-19 vaccines after reports of adverse events among adult recipients of Janssen (Johnson \& Johnson) and mRNA COVID-19 vaccines (Pfizer-BioNTech and Moderna): Update from the Advisory Committee on Immunization Practices - United States, July 2021. MMWR Morb Mortal Wkly Rep 2021;70:1094-9.

6. Barda N, Dagan N, Ben-Shlomo Y, et al. Safety of the BNT162b2 mRNA Covid-19 vaccine in a nationwide setting. N Engl J Med 2021;385:1078-90.

7. Tan JTC, Tan C, Teoh J, et al. Adverse reactions and safety profile of the mRNA COVID-19 vaccines among Asian military personnel. Ann Acad Med Singap 2021;50:827-37.

8. Oster M, CDC COVID-19 Vaccine Task Force. Overview of Myocarditis and Pericarditis. Available at: https://www.cdc.gov/ vaccines/acip/meetings/downloads/slides-2021-06/02-COVIDOster-508.pdf, 23 June 2021. Accessed on 11 September 21.

9. Ministry of Health, Israel. Surveillance of myocarditis (inflammation of the heart muscle) cases between December 2020 and May 2021 (including). Available at: https:/www.gov.il/en/departments/ news/01062021-03, 2 June 2021. Accessed on 11 September 2021.

10. Diaz GA, Parsons GT, Gering SK, et al. Myocarditis and pericarditis after vaccination for COVID-19. JAMA 2021;326:1210-2.

11. Bozkurt B, Kamat I, Hotez PJ. Myocarditis with COVID-19 mRNA vaccines. Circulation 2021;144:471-84.

12. Caso F, Costa L, Ruscitti P, et al. Could Sars-coronavirus-2 trigger autoimmune and/or autoinflammatory mechanisms in genetically predisposed subjects? Autoimmun Rev 2020;19:102524

13. Vojdani A, Kharrazian D. Potential antigenic cross-reactivity between SARS-CoV-2 and human tissue with a possible link to an increase in autoimmune diseases. Clin Immunol 2020;217:108480.

14. Fairweather D, Cooper LT Jr, Blauwet LA. Sex and gender differences in myocarditis and dilated cardiomyopathy. Curr Probl Cardiol 2013;38:7-46.

15. Ministry of Health, Singapore. Child Vaccination. Available at: https://www.moh.gov.sg/covid-19/vaccination/faqs---children-relatedvaccination-matters. Accessed on 11 September 2021.

16. Husby A, Hansen JV, Fosbøl E, et al. SARS-CoV-2 vaccination and myocarditis or myopericarditis: population based cohort study. BMJ 2021;375:e068665. 